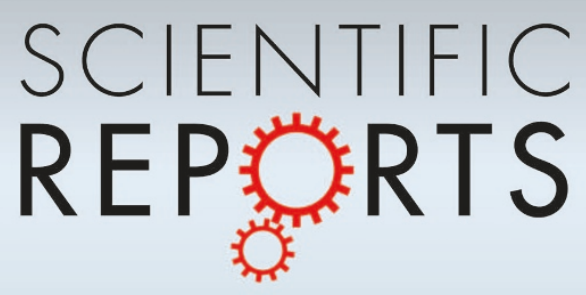

OPEN

SUBJECT AREAS:

APPLIED PHYSICS

MICRO-OPTICS

NANOPHOTONICS AND PLASMONICS

Received

3 December 2013

Accepted

20 May 2014

Published

24 June 2014

Correspondence and requests for materials should be addressed to

J.W. (jwang@hust. edu.cn)

\section{Optically-controlled extinction ratio and Q-factor tunable silicon microring resonators based on optical forces}

\author{
Yun Long \& Jian Wang
}

Wuhan National Laboratory for Optoelectronics, School of Optical and Electronic Information, Huazhong University of Science and Technology, Wuhan 430074, Hubei, China.

Tunability is a desirable property of microring resonators to facilitate superior performance. Using light to control light, we present an alternative simple approach to tuning the extinction ratio (ER) and Q-factor of silicon microring resonators based on optical forces. We design an opto-mechanical tunable silicon microring resonator consisting of an add-drop microring resonator and a control-light-carrying waveguide ("controlling" waveguide). One of the two bus waveguides of the microring resonator is a deformable nanostring put in parallel with the "controlling" waveguide. The tuning mechanism relies on the optical force induced deflection of suspended nanostring, leading to the change of coupling coefficient of microring and resultant tuning of ER and Q-factor. Two possible geometries, i.e. double-clamped nanostring and cantilever nanostring, are studied in detail for comparison. The obtained results imply a favorable structure with the microring positioned at the end of the cantilever nanostring. It features a wide tuning range of ER from 5.6 to $39.9 \mathrm{~dB}$ and Q-factor from 309 to 639 as changing the control power from 0 to $1.4 \mathrm{~mW}$.

ilicon photonics has become one of the most promising photonic integration platforms in the recent years owing to its small footprint, reduced power consumption, and availability of complementary metal-oxidesemiconductor (CMOS) fabrication technology ${ }^{1-6}$. The attractive small footprint feature of silicon waveguide structures is because of the high refractive index contrast between silicon and its oxide or air, which enables tight light confinement and benefits small bending radius of waveguide. Because of its unprecedented small size for potential high-density photonic interaction, silicon microring resonator, i.e. compact bended silicon waveguide in a small loop configuration, is of great importance to accelerate the success of silicon photonics ${ }^{7}$. Silicon microring resonators have been employed to facilitate miscellaneous applications, such as sensors ${ }^{8}$, modulators ${ }^{9}$, optical switching $^{10}$, optical data format conversion ${ }^{11}$, and on-chip optical interconnects ${ }^{12}$.

Generally speaking, all the optical properties of a passive silicon microring resonator, e.g. resonance wavelength, free spectral range, extinction ratio (ER), quality factor (Q-factor), are predetermined by the structure design and thus fixed once fabricated. In particular, it is always common to see the offset between the actually achieved properties and preliminarily designed ones due to the unavoidable fabrication errors. Hence, tunability of microring resonators is highly desirable in practical applications to facilitate superior performance. Previous tuning operations were mostly achieved by changing the refractive index of the materials of microring resonators. The already presented tuning mechanisms included the use of free-carrier dispersion effect ${ }^{9}$ and thermo-optic effect ${ }^{13}$. The free carriers and the thermal nonlinear optical effect required for tuning can be generated either electrically or optically, enabling different applications including electro-optic modulators, optical switches, optical routers, optical logic functions, and optical memories ${ }^{14}$. Most of these works focused on the resonance tuning. However, so far there have been limited research efforts on tuning the ER and Q-factor of microring resonators.

Recently, optical forces have attracted significant interest, especially in the areas of nanoparticle manipulation ${ }^{15}$ and opto-mechanically driven nanostructures ${ }^{16-23}$. One interesting example was a broadband opto-mechanical phase shifter for photonic integrated circuits, which was enabled by optical force ${ }^{20}$. It is noted that optical force also provides a possible solution to achieve optically-controlled tuning of photonic devices by opto-mechanical displacement. In this scenario, a laudable goal would be to flexibly tune the optical properties of silicon microring resonators (e.g. ER, Q-factor) using optical forces ${ }^{24}$.

In this paper, we propose an alternative approach to tuning the ER and Q-factor of silicon microring resonators by optical forces. Two opto-mechanical structures (double-clamped and cantilever) are studied for comparison. A 
wide tuning range of ER from 5.6 to $39.9 \mathrm{~dB}$ and Q-factor from 309 to 639 is achievable. Only $1.4 \mathrm{~mW}$ control light is required for the cantilever structure.

\section{Results}

Concept and principle of opto-mechanical tunable microring resonators. Figure 1 depicts the concept, 3D structure and operation principle of the designed optically-controlled (i.e. optomechanical) tunable silicon microring resonator. It consists of a control-light-carrying waveguide (named "controlling" waveguide) and an add-drop microring resonator with a radius of $3 \mu \mathrm{m}$. One of the two bus waveguides of the microring resonator is a deformable nanostring put in parallel with the "controlling" waveguide. Since the nanostring can be deflected by the optical force between the "controlling" waveguide and nanostring, the separation and resultant coupling efficiency between the nanostring and microring can be adjusted optically by varying the power of control light launched into the "controlling" waveguide. The coupling efficiency impacts on the ER and Q-factor. Hence, optically-controlled ER and Q-factor tunable microring resonators are available using optical forces. Figure 1(a)-(c) show two possible geometries, where the deformable nanostring can be a double-clamped beam or a nano- cantilever. And for the nano-cantilever scheme, two possible cases are shown. In the case 1, the ring is placed in the middle of the cantilever nanostring. In the case 2 , the ring is positioned at the end of the cantilever nanostring.

Mode distribution and optical force. In the following studies, the wavelengths of the control light and signal light are $\lambda_{c}=1550 \mathrm{~nm}$ and $\lambda_{s}=1543 \mathrm{~nm}$, respectively. The cross section and mode distribution of the combined "controlling" waveguide and nanostring are shown in Fig. 2(a). The height and width are both $500 \mathrm{~nm}$ for the "controlling" waveguide, while 500 and $250 \mathrm{~nm}$ for the nanostring. The width of nanostring is much thinner than the "controlling" waveguide, thus the nanostring is easier to be deflected. When a control light is fed into the "controlling" waveguide, optical force is formed between the "controlling" waveguide and nanostring to attract and deform the nanostring, resulting in a varied separation between the "controlling" waveguide and nanostring as well as between the nanostring and microring. Note that the effective refractive index of combined "controlling" waveguide and nanostring changes with varied separation between them, which can be calculated by the finite element method. With the obtained separation-dependent effective refractive index, optical force can

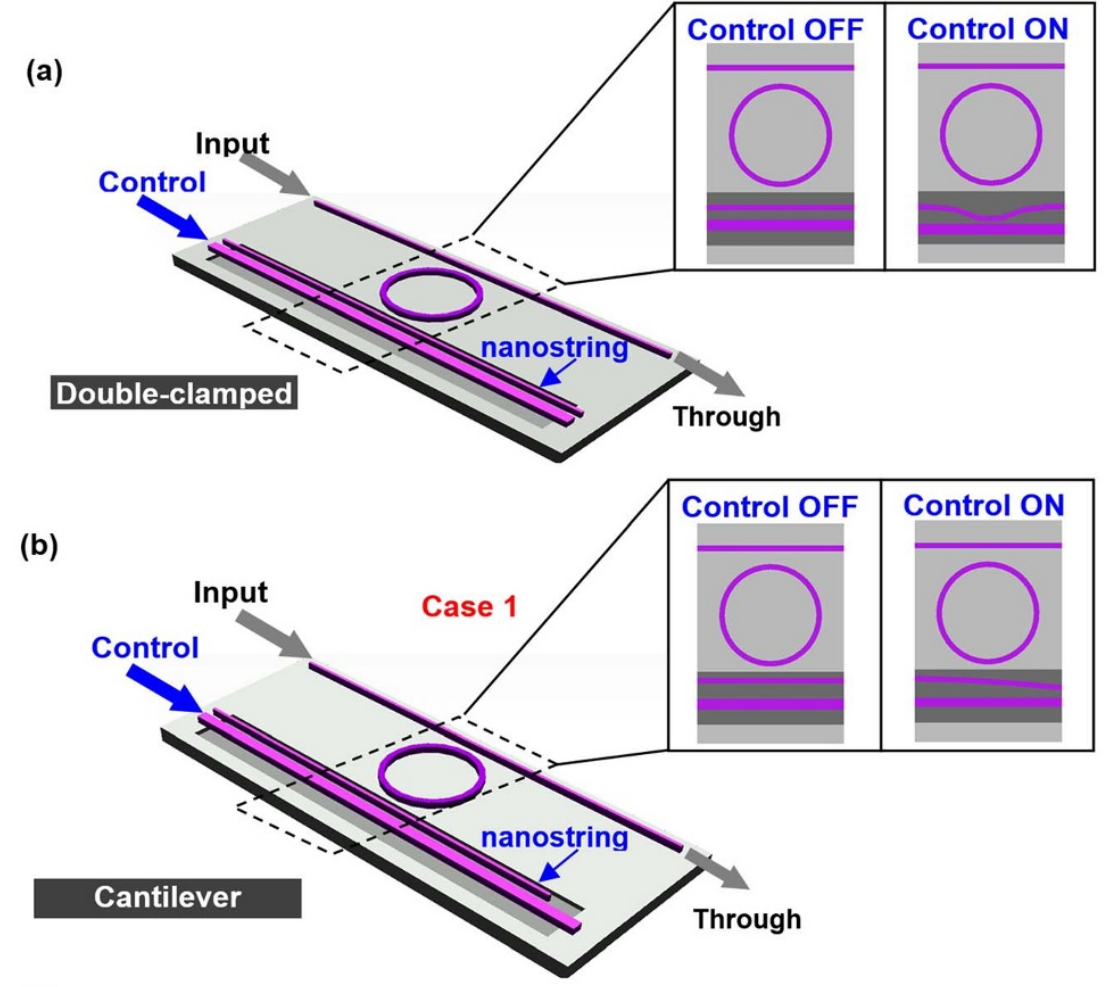

(c)

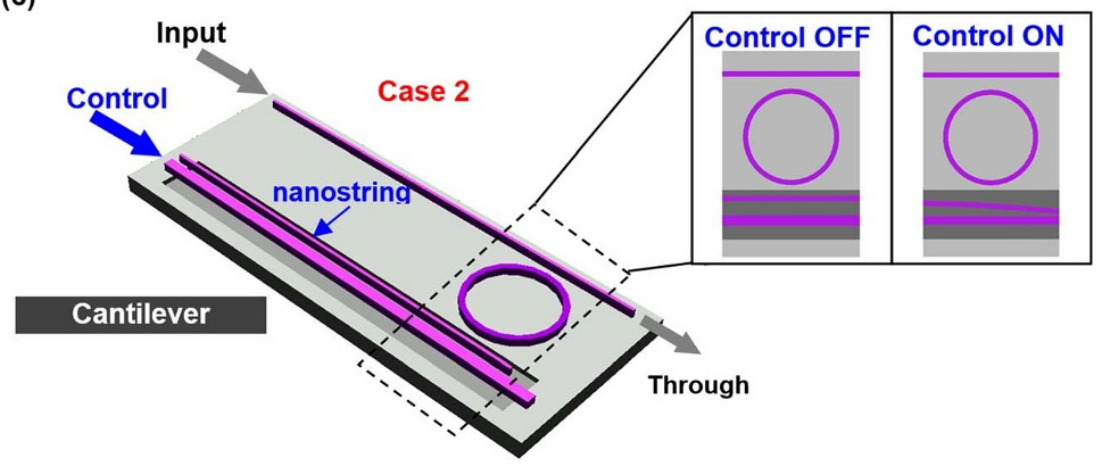

Figure 1 Schematic illustration of optically-controlled tunable silicon microring resonators. (a) The nanostring is double-clamped. (b)(c) The nanostring is a cantilever. (b) Case 1: microring in the middle of cantilever nanostring. (c) Case 2: microring at the end of cantilever nanostring. 

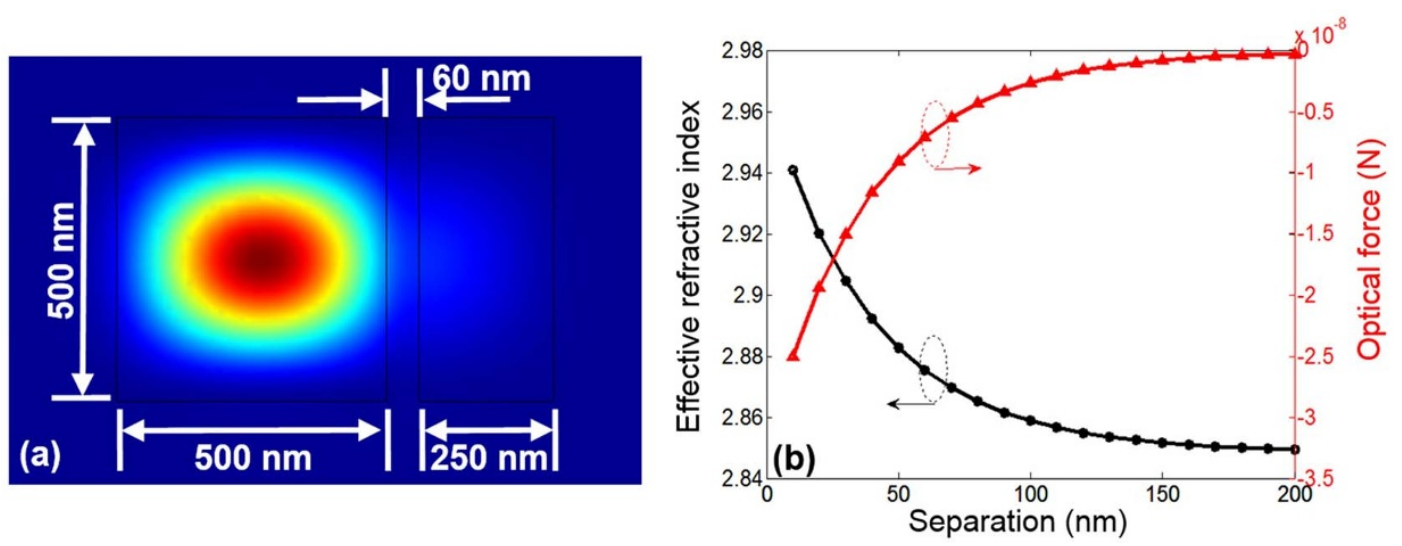

Figure $2 \mid$ (a) Cross section and mode distribution. (b) Effective refractive index and optical force versus separation between "controlling" waveguide and nanostring.

be derived from the optical force equation ${ }^{21}$. Figure $2(\mathrm{~b})$ plots the calculated effective refractive index and optical force as a function of the separation between the "controlling" waveguide and nanostring. Moreover, the deflection equation is deduced according to the EulerBernoulli beam theory ${ }^{20}$. By solving the equations for optical force and deflection, we can calculate the deflection of "controlling" waveguide and nanostring under two cases of boundary conditions for nanostring, i.e. double-clamped and cantilever.

Deflection of double-clamped nanostring and nano-cantilever. Figure 3(a) shows calculated deflection of "controlling" waveguide and double-clamped nanostring under a nanostring length of $\mathrm{L}=$ $60 \mu \mathrm{m}$ and different control powers. The higher the control power launched, the larger the deflection achieved. The maximum deflection happens in the middle of the double-clamped nanostring. For the nano-cantilever, the deflection is shown in Fig. 3(b). It is found that the desired control power can be more than one order of magnitude lower compared to double-clamped case to achieve the same level deflection. Also, the deflection increases along the nanostring.

Performance of opto-mechanical tunable microring resonators. To illustrate the phenomenon of opto-mechanical tunable microring resonators, a signal light is launched into the structure from the input port as marked in Fig. 1. Figure 4 shows an example which presents a $2 \mathrm{D}$ sketch map of optically-controlled microring resonator using a $2 \mathrm{D}$ finite-difference time-domain (2D-FDTD) method. Both scenarios in Fig. 4(a) and (b) are on resonance. Figure 4(a) corresponds to a low ER in the absence of control light.

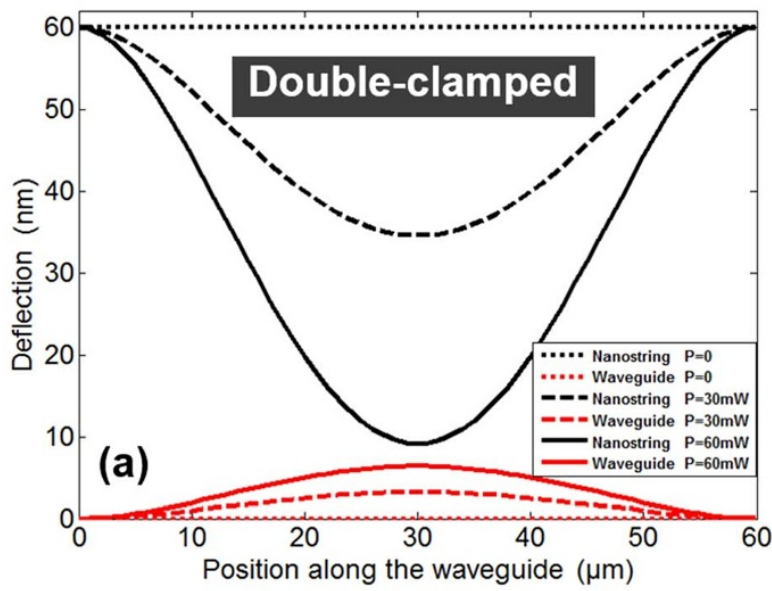

By contrast, Figure 4(b) corresponds to a high ER in the present of control light and resultant optical force.

Figure 5(a), (c) and (e) depict the transmission spectra (through port as marked in Fig. 1) under different control powers for three types of structures corresponding to Fig. 1(a), (b) and (c), i.e. doubleclamped nanostring, case 1 of cantilever nanostring, and case 2 of cantilever nanostring. The coupling efficiency between the nanostring and microring is calculated by a 3D-FDTD numerical computation. A transmission matrix method is then used to calculate the transmission spectra ${ }^{25}$. The coupling efficiency between the other bus waveguide (from input to through ports as marked in Fig. 1) and the microring is fixed at 0.19 . The loss coefficient of the ring is chosen to be 0.99 inside the microring. The initial separation (in the absence of control light) between the "controlling" waveguide and nanostring is $60 \mathrm{~nm}$. As shown in Fig. 5(a), (c) and (e), one can clearly see the varied ER and Q-factor by changing the control power, which is because of optical force-induced mechanical deflection and resultant change of coupling efficiency between the nanostring and microring. Moreover, for double-clamped nanostring shown in Fig. 5(a), relatively large control power $(60 \mathrm{~mW})$ is required to achieve large deflection and resultant large change of ER and Q-factor. For the case 1 of cantilever nanostring, a low control power $(1.4 \mathrm{~mW})$ can even drive the deflection to tune the ER and Q-factor, as shown in Fig. 5(c). However, limited change of ER and Q-factor is available as putting the microring in the middle of the cantilever nanostring. For the case 2 of cantilever nanostring with the microring positioned at the end of the cantilever nanostring, the same low control power

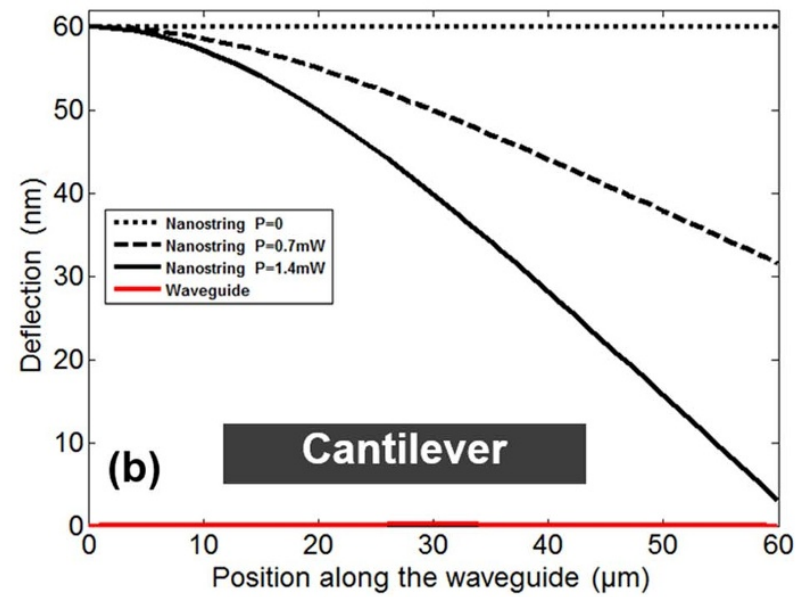

Figure 3| (a) Deflection of "controlling" waveguide and nanostring along the waveguide/nanostring under a length of $60 \mu \mathrm{m}$ and different control powers (P). (a) Double-clamped nanostring. (b) Cantilever nanostring. 

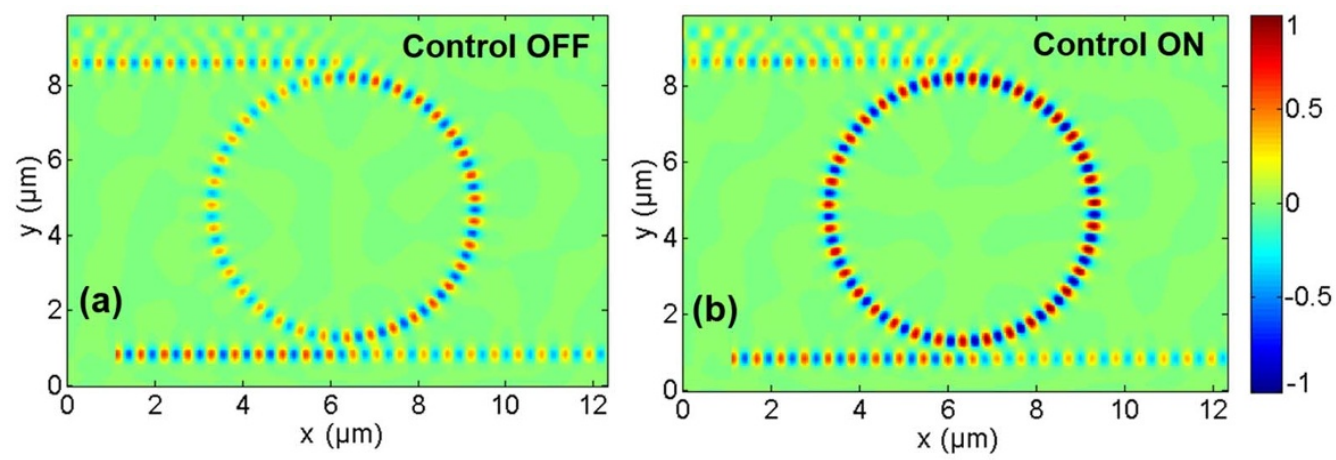

Figure $4 \mid$ Field distributions of microring resonators. (a) Low ER in the absence of control light. (b) High ER in the presence of control light.
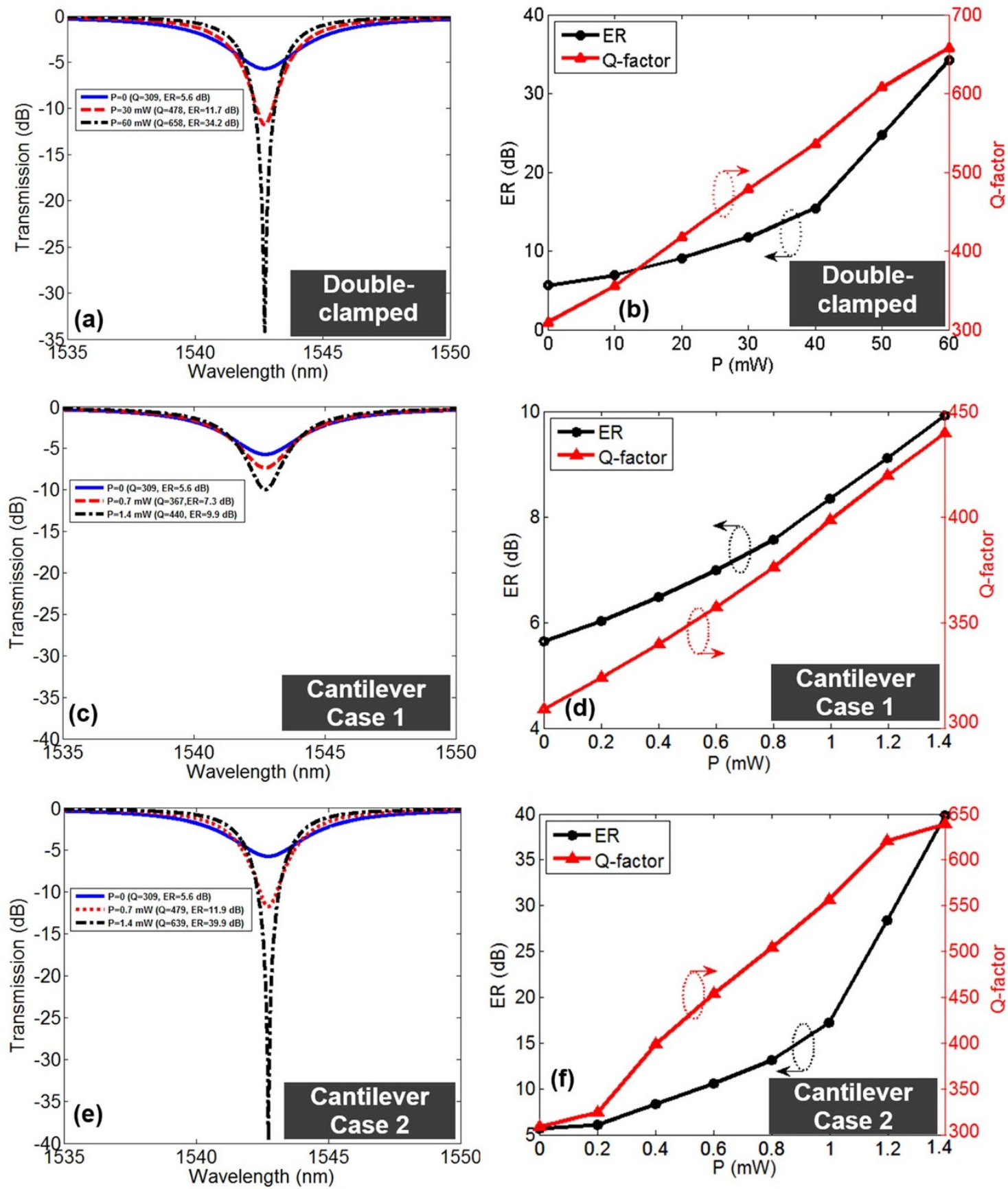

Figure $5 \mid(a)(c)(e)$ Transmission spectra under different control powers. (b)(d)(f) ER and Q-factor versus control power. (a)(b) Double-clamped nanostring. (c)(d) Case 1 of cantilever nanostring. (e)(f) Case 2 of cantilever nanostring. 
(1.4 mW) can enable significant change of ER and Q-factor. The interesting phenomena shown in Fig. 5(c) and (e) can be briefly explained with the fact that the microring close to the end of cantilever nanostring can feel larger deflection of nanostring, leading to larger variation of coupling efficiency between nanostring and microring and resultant larger change of ER and Q-factor. Actually, when moving the microring from the start to the end of the cantilever nanostring, it is expected that the change of ER and Qfactor increases correspondingly.

Figure 5(b), (d) and (f) further show the ER and Q-factor as a function of control power for double-clamped nanostring, case 1 and case 2 of cantilever nanostring, respectively. For the doubleclamped nanostring shown in Fig. 5(b), wide tuning ranges of ER from 5.6 to $34.2 \mathrm{~dB}$ and Q-factor from 309 to 658 are achieved as adjusting the control power from 0 to $60 \mathrm{mw}$. For the case 1 of cantilever nanostring shown in Fig. 5(d) with the microring in the middle of cantilever nanostring, tunable ER from 5.6 to $9.9 \mathrm{~dB}$ and Q-factor from 309 to 440 are obtained when changing the control power from 0 to $1.4 \mathrm{~mW}$. For the case 2 of cantilever nanostring shown in Fig. 5(f) with the microring moving to the end of cantilever nanostring, by varying the control power from 0 to $1.4 \mathrm{~mW}$, the tuning ranges of ER and Q-factor are effectively increased with the achievable ER from 5.6 to $39.9 \mathrm{~dB}$ and Q-factor from 309 to 639. In particular, by comparing the double-clamped nanostring in Fig. 5(a) and (b) with the case 2 of cantilever nanostring in Fig. 5(e) and (f), one can clearly see that the case 2 of cantilever nanostring offers superior tuning performance, i.e. more than one-order of magnitude lower control power is required to enable comparable tuning range of $\mathrm{Q}$-factor and even wider tuning range of ER.

\section{Discussion}

Optical force provides an alternative approach to facilitating the tunability of microring resonators. Benefiting from the flexibility of optical forces, optically-controlled ER and Q-factor tunable silicon microring resonators are proposed and simulated. The designed opto-mechanical tunable silicon microring resonator can be regarded as an add-drop microring resonator with one of its bus waveguides replaced by a deformable nanostring put in parallel with another control-light-carrying bus waveguide. The tuning mechanism relies on the optical force enabled deflection of nanostring. Optically-controlled deflection changes the separation and coupling efficiency between the nanostring and microring and therefore enables the tuning of ER and Q-factor. Double-clamped nanostring and two cases of cantilever nanostring are studied for comparison. The cantilever nanostring with the microring positioned at the end of the nanostring features a wide tuning range of ER from 5.6 to $39.9 \mathrm{~dB}$ and Q-factor from 309 to 639 by varying the control power from 0 to $1.4 \mathrm{~mW}$.

Optical force assisted tunability helps to adjust the optical properties of already fabricated microring resonators, e.g. achieving high ER and Q-factor. One distinct feature of the device is to use light to control light, which may find wide applications in optical signal processing, such as all-optical modulation, optical switching, optical routing, etc. One desirable goal of tunable optomechanical silicon microring resonator would be low-power consumption for on-chip applications. To further reduce the required control power, we may employ a longer nanostring which enables a larger deflection under a fixed control power. In addition, narrower nanostring is also expected to be beneficial to reduce the desired control power.

In the proposed optically-controlled ER and Q-factor tunable silicon microring resonator, the microring and one bus waveguide fixed to the substrate are nondeformable, the thicker "controlling" waveguide is double clamped with negligible deformation, and the other thinner bus waveguide, i.e. double-clamped or cantilever nanostring is deformable. As discussed in Ref. 22, in such an optomechanical system, the stability is a kind of "opto-mechanical equilibrium" which is referred to an equilibrium between an optical force and a mechanical force. The stable conditions or equilibrium positions of the system are set by the balance between the force due to the optical fields and the forces that are purely mechanical in origin. For different power of incident control light producing different optical force, the balanced mechanical force from deformation decides the degree of mechanical deformation, setting the corresponding stable conditions. For the proposed opto-mechanical system governed by "opto-mechanical equilibrium" stable conditions, one distinct feature is that the system stability is power dependent.

1) Stable power of incident control light enables a stable optomechanical system. The power fluctuation of incident control light may influence the stability of the opto-mechanical system, causing the variation of extinction ratio and Q-factor. Hence, the proposed opto-mechanical system with a high stability is achievable by employing stable control light source with ultralow level output power fluctuation.

2) Despite many mechanical degrees of freedom, only thinner double-clamped or cantilevel nanostring is deformable and the deformation of thicker "controlling" waveguide is negligible. The power of input signal light is also assumed to be much lower than the control light. These have negligible impacts on the stability of the opto-mechanical system.

Consequently, the power stability of incident control light source is the key factor affecting the stability of the proposed optomechanical system. Moreover, the dynamics of the proposed opto-mechnical system is decided by the available mechanical deformation. For instance, dynamic range of incident control power which is dependent on the initial geometric structure parameters, determines the achievable tuning range of extinction ratio and Q-factor.

In addition to "opto-mechanical equilibrium", as discussed in Ref. 22 and 23, it is also possible to achieve an equilibrium just by using optical forces alone, so called "optical equilibrium". Unlike "opto-mechanical equilibrium" where the stable conditions or equilibrium positions depend on the power of incident light, in "optical equilibrium" systems, the stable conditions or equilibrium positions are independent of the power of the incident light. The "optical equilibrium" stable conditions may enable self-tuned and self-stablized optical circuits. In this scenario, further improvement of the proposed opto-mechanical tunable silicon microring resonator with "optical equilibrium" could be considered to facilitate compact and controllable devices with novel functionalities.

The designed ER and Q-factor tunable silicon microring resonators might be fabricated using similar fabrication processes as reported in recent opto-mechanical devices ${ }^{26-29}$. All the structures can be fabricated on a standard silicon-on-insulator (SOI) wafer with a silicon layer and a buried oxide layer. Firstly, the patterns of device structures are written by electron-beam lithography (EBL) or deep ultraviolet (DUV) lithography and transferred to the silicon layer using inductively coupled plasma reactive-ion etching (ICP-RIE). Secondly, plasma enhanced chemical vapor deposition (PECVD) process is followed to deposit an upper cladding $\mathrm{SiO}_{2}$ layer to cover silicon microring resonators. Thirdly, after patterning the upper $\mathrm{SiO}_{2}$ layer, the window areas directly above the free-hanging parts are etched away through dry etching. Finally, selective etching process using buffered oxide etching (BOE) solution is employed to remove the buried oxide layer underneath the free-hanging parts. Consequently, the movable free-hanging parts of the device structures are released and the designed tunable opto-mechnical silicon microring resonators are fabricated. 


\section{Methods}

Microring resonators. The ER of microring resonators is expressed by

$$
\begin{aligned}
E R & =10 \log _{10}\left[\frac{\left(\sqrt{1-\kappa_{1}^{2}}+a \sqrt{1-\kappa_{2}^{2}}\right)\left(1-a \sqrt{1-\kappa_{1}^{2}} \sqrt{1-\kappa_{2}^{2}}\right)}{\left(\sqrt{1-\kappa_{1}^{2}}-a \sqrt{1-\kappa_{2}^{2}}\right)\left(1+a \sqrt{1-\kappa_{1}^{2}} \sqrt{1-\kappa_{2}^{2}}\right)}\right]^{2} \\
& =10 \log _{10}\left[\frac{\left(r_{1}+a r_{2}\right)\left(1-a r_{1} r_{2}\right)}{\left(r_{1}-a r_{2}\right)\left(1+a r_{1} r_{2}\right)}\right]^{2}
\end{aligned}
$$

where $a$ is the field round-trip loss coefficient of the ring (zero loss: $a=1$ ). $\kappa_{1}$ and $\kappa_{2}$ are the field coupling coefficients through the couplers at input port and add-drop port, respectively. $r_{1}$ and $r_{2}$ are the field transmission coefficients through the couplers at input port and add-drop port, respectively. The transmission coefficient $r_{i}(i=1,2)$ is related to the coupling coefficient $\kappa_{i}$ through $r_{i}=\sqrt{1-\kappa_{i}^{2}}$ without considering the loss of coupling regions.

The Q-factor of microring resonators is calculated by

$$
Q=\frac{\pi n_{e f f} L_{c}}{\lambda} \frac{\sqrt{a r_{1} r_{2}}}{1-a r_{1} r_{2}}
$$

where $L_{c}$ is the circumference of microring given by $L_{c}=2 \pi R$ ( $R$ : radius of microring). $n_{e f f}$ is the effective refractive index of the waveguide mode of interest. $\lambda$ is the wavelength in vacuum.

According to Eq. (1), an add-drop microring resonator can offer a maximal ER as the critical coupling condition $r_{1}=a r_{2}$ is satisfied. The tuning mechanism of the designed tunable opto-mechnical silicon microring resonators is to change $r_{2}$ through optical force induced deflection of nanostring. Based on Eqs. (1) and (2), the change of $r_{2}$ enables the tuning of ER and Q-factor. It is expected that in principle one can achieve maximal ER by precisely adjusting the control power to meet the critical coupling condition $\left(r_{1}=a r_{2}\right)$.

Optical force. Generally speaking, one should use Maxwell stress tensor method to accurately calculate the optical forces in the proposed opto-mechanical system ${ }^{30,31}$. However, considering the complexity of the proposed opto-mechanical system, a full numerical approach using Maxwell stress tensor method is computationally intensive. Recently, an analytical formalism known as Response Theory of Optical Forces (RTOF) was presented for the treatment of optical forces in opto-mechanical systems, providing a very unique and powerful analytical alternative to Maxwell stress tensor methods ${ }^{21}$. Perfect agreement is proved between the RTOF method and Maxwell stress tensor method. Following the RTOF method, the optical force near a waveguide takes the form ${ }^{21}$

$$
F=\frac{L P}{c} \frac{\partial n_{e f f}}{\partial d}
$$

where $L$ is the length of the waveguide. $d$ is the gap between the waveguide and nanostring. $P$ is the input power of light. $c$ is the speed of light in vacuum.

The effective refractive index $n_{\text {eff }}$ as a function of the gap $d$ can be solved by the finite element method, and then the optical force can be calculated based on Eq. (3).

Optical force induced deflections. Deflections of both waveguide and nanostring are determined by Euler-Bernoulli beam theory ${ }^{20}$

$$
\frac{d^{4} u}{d z^{4}}=\frac{12}{E t^{2}} \cdot \frac{F}{L P} \cdot \frac{P}{A}
$$

where $E=131 \times 10^{9} \mathrm{~Pa}$ is the Young's modulus of silicon. $t$ is the width of the waveguide (nanostring). $A$ is the geometric area of the waveguide (nanostring) cross section.

To solve the above fourth-order differential equation, one needs to know the boundary conditions. For the nanostring, there are two kinds of geometric structure, i.e. double-clamped and cantilever, corresponding to two types of boundary conditions. For the double-clamped nanostring, both the waveguide and the nanostring satisfy the same boundary conditions of $u(0)=\frac{d u}{d z}(0)=u(L)=\frac{d u}{d z}(L)=0$.

By combining Eqs. (3) and (4) together with boundary conditions, we can analytically derive optical force induced deflections of both waveguide and nanostring expressed by

$$
u=\frac{1}{2} \frac{F}{A L t^{2} E} z^{4}-\frac{F}{A t^{2} E} z^{3}+\frac{1}{2} \frac{F L}{A t^{2} E} z^{2}
$$

For the cantilever nanostring, the waveguide still obey the same boundary conditions of $u(0)=\frac{d u}{d z}(0)=u(L)=\frac{d u}{d z}(L)=0$, while the boundary conditions for the cantilever nanostring become $u(0)=\frac{d u}{d z}(0)=\frac{d^{2}(u)}{d^{2} z}(L)=\frac{d^{3} u}{d^{3} z}(L)=0$. Using Eqs. (3) and (4) together with cantilever nanostring boundary conditions, we can analytically deduce the deflection of the cantilever nanostring written by

$$
u=\frac{1}{2} \frac{F}{A L t^{2} E} z^{4}-2 \frac{F}{A t^{2} E} z^{3}+3 \frac{F L}{A t^{2} E} z^{2}
$$

1. Bogaerts, W. et al. Nanophotonic waveguides in silicon-on-insulator fabricated with CMOS technology. J. Lightwave Technol. 23, 401-412 (2005).

2. Soref, R. A. The Past, Present, and Future of Silicon Photonics. IEEE J. Sel. Top. Quantum Electron. 12, 1678-1687 (2006).

3. Little, B. E., Chu, S. T., Pan, W. \& Kokubun, Y. Microring resonator arrays for VLSI photonics. IEEE Photon. Technol. Lett. 12, 323-325 (2000).

4. Little, B. E. et al. Ultra-compact $\mathrm{Si}-\mathrm{SiO} 2$ microring resonator optical channel dropping filters. IEEE Photon. Technol. Lett. 10, 549-551 (1998).

5. Soref, R. A., Emelett, S. J. \& Buchwald, W. R. Silicon waveguided components for the long-wave infrared region. J. Opt. A, Pure Appl. Opt. 8, 840-848 (2006).

6. Lee, B. G. et al. Ultrahigh-bandwidth silicon photonic nanowire waveguides for on-chip networks. IEEE Photon. Technol. Lett. 20, 398-400 (2008).

7. Bogaerts, W. et al. Silicon microring resonators. Laser \& Photonics Rev. 6, 47-73 (2012).

8. Yi, H., Citrin, D. S. \& Zhou, Z. Highly sensitive silicon microring sensor with sharp asymmetrical resonance. Opt. Express 18, 2967-2972 (2010).

9. Almeida, V. R. \& Lipson, M. Optical bistability on a silicon chip. Opt. Lett. 29, 2387-2389 (2004).

10. Xu, Q., Schmidt, B., Pradhan, S. \& Lipson, M. Micrometre-scale silicon electrooptic modulator. Nature 435, 325-327 (2005).

11. Ding, Y. et al. Multi-channel WDM RZ-to-NRZ format conversion at $50 \mathrm{Gbit} / \mathrm{s}$ based on single silicon microring resonator. Opt. Express 18, 21121-21130 (2010).

12. Little, B. E. et al. Microring resonator channel dropping filters. J. Lightwave Technol. 15, 998-1005 (1997).

13. Almeida, V. R. et al. All-optical switching on a silicon chip. Opt. Lett. 29, 2867-2869 (2004).

14. Chremmos, I., Uzunoglu, N. \& Schwelb, O. Photonic microresonator research and applications (Springer, 2010).

15. Chu, S. Laser Manipulation of Atoms and Particles. Science 253, 961-866 (1991).

16. Wiederhecker, G. S., Chen, L., Gondarenko, A. \& Lipson, M. Controlling photonic structures using optical forces. Nature 462, 633-636 (2009).

17. Povinelli, M. et al. High-Q enhancement of attractive and repulsive optical forces between coupled whispering-gallery- mode resonators. Opt. Express 13, 8286-8295 (2005).

18. Li, M. et al. Harnessing optical forces in integrated photonic circuits. Nature 456, 480-484 (2008)

19. Fong, K. Y. et al. Tunable optical coupler controlled by optical gradient forces. Opt. Express 19, 15098-15108 (2011).

20. Guo, X. et al. Broadband opto-mechanical phase shifter for photonic integrated circuits. Appl. Phys. Lett. 101, 071114 (2012).

21. Rakich, P. T., Popovic, M. A. \& Wang, Z. General treatment of optical forces and potentials in mechanically variable photonic systems. Opt. Express 17, 18116-18135 (2009).

22. Intaraprasonk, V. \& Fan, S. A lateral optical equilibrium in waveguide-resonator optical force. Opt. Express 21, 25257-25270 (2013).

23. Intaraprasonk, V. \& Fan, S. Optical pulling force and conveyor belt effect in resonator-waveguide system. Opt.Lett. 38, 3264-3267 (2013).

24. Long, Y. et al. Optically-controlled extinction ratio and Q-factor tunable silicon microring resonators based on optical forces, in Proceedings of Photonics Conference (IPC), 2013 IEEE paper TuH3.5, Bellevue Washington (IEEE Photonics Society, 2013).

25. Schwelb, O. Transmission, group delay, and dispersion in single-ring optical resonators and add/drop filters-a tutorial overview. J. Lightwave Technol. 22, 1380-1394 (2004).

26. Cai, H. et al. Nano-opto-mechanical actuator driven by gradient optical force. Appl. Phys. Lett. 100, 013108 (2012).

27. Deotare Parag, B. et al. All optical reconfiguration of optomechanical filters. Nat. Commun. 3, 846 (2012).

28. Yu, Y. F. et al. Force-induced optical nonlinearity and Kerr-like coefficient in opto-mechanical ring resonators. Opt. Express 20, 18005-18015 (2012).

29. Eichenfield Matt. et al. A picogram-and nanometre-scale photonic-crystal optomechanical cavity. Nature 459, 550-555 (2009).

30. Dienerowitz, M. et al. Optical manipulation of nanoparticles: a review. J. Nanophoton. 2, 021875 (2008).

31. Gordon, J. P. Radiation forces and momenta in dielectric media. Phys. Rev. A 8 , 14-21 (1973)

\section{Acknowledgments}

This work was supported by the National Natural Science Foundation of China (NSFC) under grants 61222502, 61077051, 11274131 and L1222026, the National Basic Research Program of China (973 Program) under grant 2014CB340004, the Program for New Century Excellent Talents in University (NCET-11-0182), the Wuhan Science and Technology Plan Project under grant 2014070404010201, the Fundamental Research 
Funds of the Central Universities (HUST) under grants 2012YQ008 and 2013ZZGH003, and the seed project of Wuhan National Laboratory for Optoelectronics (WNLO). The authors thank Xuhui Li, Long Zhu, Chengcheng Gui, Zhonglai Zhang, Jing Du and Shuhui Li for helpful discussions.

\section{Author contributions}

J.W. developed the concept and conceived the design. Y.L. performed the numerical simulations. Y.L. and J.W. analyzed the data. Y.L. and J.W. contributed to writing and finalizing the paper.

\section{Additional information}

Competing financial interests: The authors declare no competing financial interests.
How to cite this article: Long, Y. \& Wang, J. Optically-controlled extinction ratio and Q-factor tunable silicon microring resonators based on optical forces. Sci. Rep. 4, 5409; DOI:10.1038/srep05409 (2014).

(c) (i) $\Theta$ This work is licensed under a Creative Commons Attribution-NonCommercialNoDerivs 4.0 International License. The images or other third party material in this article are included in the article's Creative Commons license, unless indicated otherwise in the credit line; if the material is not included under the Creative Commons license, users will need to obtain permission from the license holder in order to reproduce the material. To view a copy of this license, visit http:// creativecommons.org/licenses/by-nc-nd/4.0/ 